\title{
Urgences
}

\section{Les lieux superposés de Richard McGuire}

\section{Thierry Groensteen}

Numéro 32, mai 1991

Lectures de bandes dessinées

URI : https://id.erudit.org/iderudit/025651ar

DOI : https://doi.org/10.7202/025651ar

Aller au sommaire du numéro

Éditeur(s)

Urgences

ISSN

0226-9554 (imprimé)

1927-3924 (numérique)

Découvrir la revue

Citer cet article

Groensteen, T. (1991). Les lieux superposés de Richard McGuire. Urgences, (32), 95-109. https://doi.org/10.7202/025651ar d'utilisation que vous pouvez consulter en ligne.

https://apropos.erudit.org/fr/usagers/politique-dutilisation/ 


\section{Les lieux superposés de Richard McGuire Thierry Groensteen}

$R a w$, la revue animée par l'artiste américain Art Spiegelman, n'a jamais cessé de créer la surprise depuis son apparition en 1980. En 1989, entrant dans le giron d'un géant éditorial, Penguin Books, elle voyait rapetisser son format, grossir sa pagination et augmenter sa diffusion. Si ces changements pouvaient faire craindre une banalisation du contenu, une histoire au moins apportait, dès le premier numéro de cette nouvelle formule, le plus éclatant des démentis.

Intitulée Here ( Ici "), cette histoire n'occupe pas plus de six pages (p. 69 à 74). L'auteur, Richard McGuire, un musicien et illustrateur new-yorkais, signe là, à ma connaissance, sa première bande dessinée. C'est par ses qualités conceptuelles, non par son graphisme, que ce bref récit retient l'attention. Et d'abord parce que, en fait de récit, il contrevient à tous les usages (figures 1-6).

Ainsi, la vignette inaugurale (un coin de pièce dont plancher, murs et fenêtre ne présentent que des surfaces également vides), loin d'accrocher l'œil ou d'introduire une action, semble, au premier regard, quasi dépourvue de signification. Dans la mesure où cet incipit ne paraît enclencher aucun procès narratif, le lecteur est conduit à supposer que sa fonction apparaîtra plus tard, sous l'effet d'une détermination rétroactive. Nous verrons, le moment venu, ce qu'il en est.

Les vignettes deux à quatre évoquent de manière elliptique, en trois flashes successifs, la naissance d'un bébé dans une famille américaine appartenant, pour ce qu'on en peut deviner, à la middle class. N'était sa parfaite banalité (qu'accusent des dialogues d'une extrême platitude), cette séquence, du moins, paraît engager le récit sur des voies plus normales. Cependant, un élément ne laisse pas d'étonner: chaque vignette porte, dans son angle supérieur gauche, la mention d'une date: 1957 . On ne peut, à cet endroit de la lecture, s'expliquer l'utilité de cette répétition. Pour ancrer la diégèse dans son temps historique, une occurrence de la date n'aurait-elle pas suffi? 
96

Les deux dernières vignettes de cette première planche, introduisant au dispositif particulier qui gouvernera ensuite le récit jusqu'à son terme, apportent rétrospectivement l'explication cherchée. L'intrigue dont nous suivions jusque-là les maigres péripéties y subit une sorte de décrochage, mimant un effet auquel l'électronique télévisuelle nous a accoutumés. L'image se réduit progressivement, découvrant ainsi derrière elle une portion de "l'écran " vignettal où une autre scène, qu'elle masque en partie, se donne à voir. Or, cette autre scène nous fait opérer un saut dans le temps. De 1957, nous sommes ramenés à 1922 dans le * fond » de la vignette 5 , puis projetés en 1971 dans celui de la vignette 6 . Ce dernier cadre accueille en outre, en son angle inférieur droit, une deuxième image incrustée, qui, elle, porte la mention " 1999 ".

On l'aura compris, la bande dessinée de Richard McGuire usera dès lors du hiatus temporel avec une implacable systématicité. Dans les cinq planches restantes, tout passage entre une vignette et la vignette consécutive équivaudra à un saut dans le passé ou dans le futur. L'instabilité de l'action est encore accrue par la multitude des incrustations, elles aussi anachroniques. Divisées en six rectangles chacune, ces six planches comportent au total, non pas trente-six, mais bien quatre-vingt-cinq vignettes, qui, à l'exception de la première, sont toutes datées 1 .

Ce qui, de la sorte, se trouve contredit, n'est rien moins que le principe fondateur de toutes les histoires en images, à savoir la coïncidence entre l'axe temporel de la consécution et l'axe logique de la conséquentialité: ce qui vient avant entraîne ou autorise ce qui vient après. En brouillant la chronologie, McGuire propose au lecteur un récit en miettes, qui n'a ni queue ni tête, et qui se dérobe à toute totalisation. Comme si l'ordre d'apparition de ces images-souvenirs était, soit volontairement anarchique, soit aléatoire.

1 Dès l'instant où l'on a compris le fonctionnement très particulier de cette bande dessinée, on revient sur son étonnement premier; l'étrange, en les vignettes 2, 3 et 4, n'était pas qu'elles fussent datées toutes les trois, mais bien que la même date fût conservée dans plusieurs vignettes consécutives. Par crainte - qu'on pourrait dire " démagogique" - de décourager d'emblée le lecteur, ou par souci "pédagogique" de favoriser un apprentissage graduel du protocole de lecture requis par ces pages, McGuire a conçu ce prologue pour ménager une voie d'accès aux pages qui suivent. 
S'il n'abandonne pas d'emblée sa lecture pour quelque divertissement moins « absurde", le lecteur hésite alors entre deux modes d'emploi de cette œuvre déconcertante qui, dérogeant à la vocation traditionnelle de la bande dessinée, ne répond pas à l'attente des amateurs de récits que nous sommes tous. Il peut y voir une sorte de jeu dont l'objet serait le rétablissement de la chronologie brouillée; c'est supputer qu'une fois les vignettes reclassées d'après leur datation, elles donneront à lire un parcours cohérent et, telles les pièces d'un puzzle, une totalité signifiante.

Ou bien, tenant pour acquis que ces pages ne recèlent aucun enjeu narratif, il peut faire le pari que ce défaut de narration (défaut au sens de manque) doit nécessairement être compensé par un gain d'un autre ordre, et se mettre dès lors en quête du protocole de lecture qui ferait apparaître cette ordonnance cachée, ce plaisir enfoui.

Qu'on m'autorise dès à présent une conclusion quelque peu anticipée: à l'épreuve, ces deux façons d'en user avec la BD de McGuire, exclusives et cependant solidaires, produisent l'une et l'autre d'intéressants résultats. Mais il m'apparaît qu'en dernier ressort, la fascination très particulière qu'exerce cette bande réside surtout dans le battement entre deux lectures qui se jouent l'une de l'autre.

La première option, celle qui consiste à rétablir les faits évoqués dans leur chronologie, se heurte à une difficulté intellectuelle. La mémoire immédiate est impuissante à engranger et à classer autant d'éclats disparates. Pour vérifier son intuition, le lecteur est donc obligé de recourir à des expédients matériels. Ce n'est que muni d'une photocopieuse, de ciseaux et d'un bâton de colle, qu'il pourra découper les quatre-vingt-cinq vignettes ${ }^{2}$ et les remonter dans le bon ordre. Ce petit travail, que j'ai accompli pour ma part, conduit aux observations suivantes.

1. Sous l'angle de la période historique couverte, le "récit" de McGuire est d'une exceptionnelle amplitude. La date la

2 Nous y renverrons dans la suite en les désignant par une suite numérique composée du numéro de la planche, en romain, suivi, en chiffres arabes, du numéro du cadre et de la date figurant dans la vignette concernée. Par exemple, III-6-1870 désignera cete portion du sixième cadre de la planche trois où paraissent un cochon et deux poules. 
98

plus reculée nous fait remonter jusqu'à l'an 500957406073 avant J.-C., tandis qu'à l'autre extrémité l'histoire s'interrompt en 2033.

2. Cette histoire est l'histoire d'un lieu. Il faut en effet supposer, sur la foi des multiples recoupements possibles et en l'absence de toute exception avérée à la règle, que l'ensemble de ces vignettes * cadrent", sous le même angle et à même distance (on parlerait au cinéma d'un plan fixe), une même portion d'espace ou un détail prélevé en son sein fragment de cette portion. À l'extrême mobilité du narrateur sur l'échelle du temps s'oppose donc l'absolue fixité du point de vue adopté. Tout se passe comme si, durant plus de cinq cents milliards d'années, un témoin immobile avait obstinément gardé l'œil rivé sur la même parcelle de notre planète.

3. En ce lieu où s'ébattaient jadis les grands sauriens, où vécurent bien plus tard des Indiens, des fermiers blancs édifièrent en 1870 un enclos, en 1901 une maison. Incendiée en 2029 , cette demeure sera démolie l'année suivante. La majorité des vignettes concernent la vie domestique des familles qui, successivement, habitèrent cette maison. De cette chronique en pointillé se détache un seul personnage stable et identifiable: William, dit «Billy", dont la naissance (en 1957) et la mort (en 2027) sont dûment signalées. Bien que je ne possède guère de renseignements biographiques sur Richard McGuire, il me parait raisonnable de supposer que Billy est son contemporain au moins approximatif, d'où, sans doute, la place privilégiée qu'il occupe dans l'économie de la bande.

4. De la vie de Billy pas plus que de celle des autres personnages représentés (tous anonymes), rien n'émerge qui paraisse digne d'intérêt. Certainement, tout cela ne valait pas la peine d'être conté. C'est bien ce dont, à sa manière, l'auteur nous avait averti en ne prenant pas la peine d'organiser cette «matière» en récit. Même rétablis dans leur chronologie, ces instantanés continuent d'être insignifiants: un verre renversé, un œil jeté sur des photos ou des films de famille, un élément de la décoration modifié, un enfant surpris en train de dérober un biscuit, mille et un propos échangés qui consternent par leur vacuité.

Ainsi, ce laborieux exercice de reconstitution a précisé les contours de la diégèse; il a permis de rétablir un ou deux 
enchaînements syntagmatiques (ainsi des trois images qui évoquent la chute du verre en 1971); mais il s'est révélé fort peu rentable en termes de scénario ou de dramaturgie ${ }^{3}$.

Il fallait pourtant cette confirmation pour lever nos derniers doutes quant aux intentions de l'auteur. Rarement pareille violence fut atteinte dans la dénonciation, en quelques pages, de la médiocrité de tant d'existences humaines. Cette chronique de la vie quotidienne, telle qu'elle est vécue dans ce lieu littéralement désigné comme un "petit coin " de l'Amérique profonde ( $c f$. vignette I-1), dresse le constat d'un mode d'existence qu'on pourrait presque qualifier de végétatif, tant il concentre les symptômes d'une condition aliénée. L'emblème de cette aliénation serait le quatrième cadre de la cinquième page, où un montage de quatre vignettes montre, dans un raccourci fulgurant, que les mêmes tâches ménagères sempiternellement recommencées donnent lieu, année après année, aux mêmes commentaires résignés.

On comprend alors qu'il importait peu de montrer tel instant plutôt que tel autre: tous les moments de semblables vies, semble nous dire cette bande, sont pareillement frappés d'insignifiance. Pour la même raison, donner à ces moments la sorte de nécessité que toute narration confêre à chacun de ses éléments constitutifs eût été une erreur. Rompre avec les conventions du récit traditionnel, c'était pour McGuire le moyen de rendre sa critique encore plus impitoyable. Ce qui était à lire n'était rien d'autre que le constat d'un vide. Ce vide dont l'incohérence du discours narratif se proposait d'emblée comme la métaphore; ce vide que résumait exemplairement la première vignette.

Mais il a été suggéré qu'une autre lecture de Here est possible. On peut en effet renoncer au confort d'une interprétation cohérente et, sans chercher secours du côté de la chronologie, s'attacher à lire les effets de sens locaux qui naissent, ponctuellement, de la contiguitté des images. Car il apparaît

3 Les deux rapprochements les plus lourds de sens (on verra que c'est beaucoup dire) sont d'abord celui, anecdotique, du chat et de la souris; ils ne se rencontraient pas sur la même page, et l'on découvre que le premier pourrait bien avoir croqué la seconde, prise au piège en 1999; ensuite, en 2030, celui, analogique, d'une vitre brisée par une balle de baseball et d'un mur percé par la boule des démolisseurs. 
vite que leur distribution dans l'espace n'est pas tout à fait désordonnée: éloignées dans le temps et sans cohérence diégétique apparente, telle paire de vignettes contiguës peut justifier sa proximité par la mise en œuvre concertée de paramètres d'un autre ordre - qui ne ressortit pas à la narration. De nombreux "micro-dialogues" se nouent ainsi entre vignettes réunies dans un même cadre ou entre cadres juxtaposés. Ces formes de coordination, participant de ce que j'ai proposé d'appeler les «effets de tressage " 4 , sont ici particulièrement variées: rime, renvoi, opposition, articulation sémantique ou symbolique. La malice est leur commun dénominateur. Sans du tout les épuiser (j’invite au contraire le lecteur à poursuivre le jeu par lui-même), j'en donnerai un échantillon.

Mes deux premiers exemples se rattachent à l'idée de l'aliénation qui était, on l'a vu, la principale conclusion à laquelle conduisait la lecture chronologique. C'est ainsi que l'inintérêt des tâches ménagères (III-4-1983) apparaît encore renforcé, par contraste, lorsqu'elles sont mises en parallèle avec un accomplissement exceptionnel, qui fait date dans l'histoire de l'humanité, comme la première mission spatiale Apollo (III-4-1966). En II-6, des femmes au physique passablement avachi bavardent avec insouciance, alors qu'au même moment (1944) de terribles événements se jouent sur la scène du monde; un tableau accroché au mur représente des vaches dans un paturage; trois petits cadres placés en incrustation (II6-1860) font défiler des animaux domestiques. La circulation qui s'établit entre ces trois compartiments de l'image induit un jugement d'équivalence entre mémères et bovidés.

Rime, reprise, dédoublement: les objets, les êtres, les sons se répondent en grand nombre. "Speak " (IV-2-1965) amène aussitôt « squeak * (IV-3-1999), et le monstrueux dinosaurien vivant en 100650010 av. J.-C. (IV-4) succède immédiatement à sa réplique miniaturisée que manipule l'enfant en l'an 2028 (IV-3).

Parce qu'elles peuvent indifféremment s'appliquer à deux situations figurées dans des cadres voisins, certaines répliques sont dotées d'un double sens qui leur confère une

4 Thierry Groensteen, « Du $7^{\circ}$ au $9^{\circ}$ art, l'inventaire des singularités ", dans CinémAction: Cinéma et bande dessinée, été 1990. 
indéniable saveur. Citons-en deux. D'abord *Billy! Put that back! ", qui s'applique d'abord au biscuit que l'enfant vient de saisir (IV-5-1968 a et b), mais où il est permis de lire une plaisante injonction à remettre en place l'arbre fraîchement scié (en 2032!) qui apparaît entre les deux protagonistes; puis, en VI-12-2027, l'énoncé « You know that guy who used to live here", mis en facteur commun pour Billy, qui fut le précédent locataire (VI-2-1987), et pour l'Indien tué en 1850 sur cette même terre dont il était alors le légitime détenteur. Chacun de ces effets de sens, établi au prix d'un court-circuit temporel, se fonde sur une configuration spatiale ou, plus justement, topologique. C'est parce que le regard du lecteur transite obligatoirement par la zone où figure l'arbre coupé que celui-ci s'interpose entre les deux termes de la scène du biscuit, imprimant au passage une signification alternative à la phrase prononcée par la mère.

La topologie est principe d'autres dialogues paradoxaux entre vignettes diégétíquement irréconciliables. En I-6, le chat de 1999 se dresse vers le biberon offert au bébé de 1957, dont il ferait volontiers son profit. En III-3-1971, le verre brisé ne tombe pas de nulle part: il amorçait sa chute sept centimètres plus haut, en III-1-1971, où le liquide qu'il renfermait était opposé aux flammes de l'incendie figuré en III-1-2029. Le personnage figé dans la position du poirier (III-3-1960) semble n'avoir d'autre fonction que de souligner, par sa verticalité orientée vers le bas, l'axe de la chute du récipient. De même, le corps représenté en III-3-1990 pointe, dans son oblicité, vers le cadre III-2, comme pour mieux amarrer ensemble deux vignettes que reliaient déjà les énoncés verbaux ( What time* et *Oh, six, six-thirty, what is it now ? ; l'échange sur ce thème commence en réalité en III-1).

La construction la plus élaborée me paraît celle proposée en VI-5. À partir d'éléments empruntés aux trois vignettes respectivement datées 1750,1986 et 2030 , une cohérence enfouie peut être mise au jour. Deux motifs circulaires sont distants d'à peine deux centimètres: la balle de baseball et le motif qui orne la tente. De même diamètre, ces deux cercles fournissent séparément les éléments du signe du Tao: une ligne sinueuse, d'une part, l'opposition entre noir et blanc, d'autre part. Ils permettent donc sa recomposition mentale par le lecteur. Or, au couple Yin-Yang correspond notamment 
la dualité masculin-féminin, et leur union dans la complémentarité. C'est bien une telle rencontre que synthétise la zone VI-5-1986, les jambes de l'homme et de la femme se trouvant d'ailleurs à la verticale des motifs circulaires.

Sans entrer plus avant dans le recensement des opérations de tressage effectuées par McGuire et des figures qu'elles dessinent, je veux insister sur les traits qui, pour moi, font de cette bande dessinée une œuvre exemplaire. Here se joue de notre attente première, et commence par décevoir le lecteur qui ne peut s'accrocher au "fil d'Ariane" d'un récit. Mais, à défaut de la linéarité syntagmatique qui prévaut dans les bandes dessinées traditionnelles, cette antihistoire fait partout fleurir des effets de sens locaux, qu'il appartient au lecteur de réaliser au prix d'une participation active. La dimension pédagogique de ces pages est irrécusable: elles réclament une attention minutieuse au moindre signe, exigent la construction et la vérification d'hypothèses, bref nous font passer d'un registre d'activité à un autre, de celui de la lecture à celui du jeu. En détricotant le tissu temporel et événementiel, en ayant en outre massivement recours à la procédure de l'incrustation, McGuire s'est donné le moyen de renforcer et de faire monter en surface des propriétés trop souvent négligées dans l'usage qu'il est fait des vignettes : leur incomplétude, leur localisation, la multiplicité de leurs niveaux de signifiance.

Des images apparemment vaines, des mises en scène oiseuses telles que cette bande en propose à foison se sémantisent obliquement (insidieusement) par le seul jeu de leur interaction, qui ne connaît pas de fin assignable. Nombre de vignettes se révèlent ainsi à double, à triple, à multiple entente, selon que, au gré des voisinages et des résurgences, on les indexe sur tel ou tel de leurs composants iconiques ou de leurs paramètres formels. Dégagée de tout assujettissement à un récit linéaire, l'histoire dessinée devient un réseau que l'on peut parcourir librement et selon toutes sortes de pistes. En intitulant son œuvre «Ici», Richard McGuire semble avoir voulu nous signifier que le sens se construit partout, c'est-àdire à quelque endroit que le lecteur soit rendu. En associant à ce titre une image emblématique du vide, il a peut-être indiqué avec un surcroît de précision que cette construction 
sémantique s'échafaude dans le «blanc * intervignettal, dans cet intervalle où s'accomplit et se dépose la pensée même d'un rapprochement.

Mais, on l'a dit, cet usage moderne et non conventionnel du dispositif compartimenté de la bande dessinée s'accommode ici d'un autre protocole de lecture, celui qui passe par la restauration de la chronologie contrariée. Le fait que chaque portion vignettale soit précisément datée invite à cette mise en ordre. Ainsi, chacun de ces cadres, chacun de ces lieux, chacun de ces "ici et maintenant * se trouve pris dans un double système de coordonnées : celui, topologique et effectif, que ces pages nous donnent à observer, et celui, chronologique et virtuel, dont les inscriptions autorisent le rétablissement. À l'épreuve, il est loisible de constater que ces deux usages ne s'annulent pas, mais se renforcent l'un l'autre, tous deux participant à l'apothéose d'une vision critique et ironique du monde. 
HERE
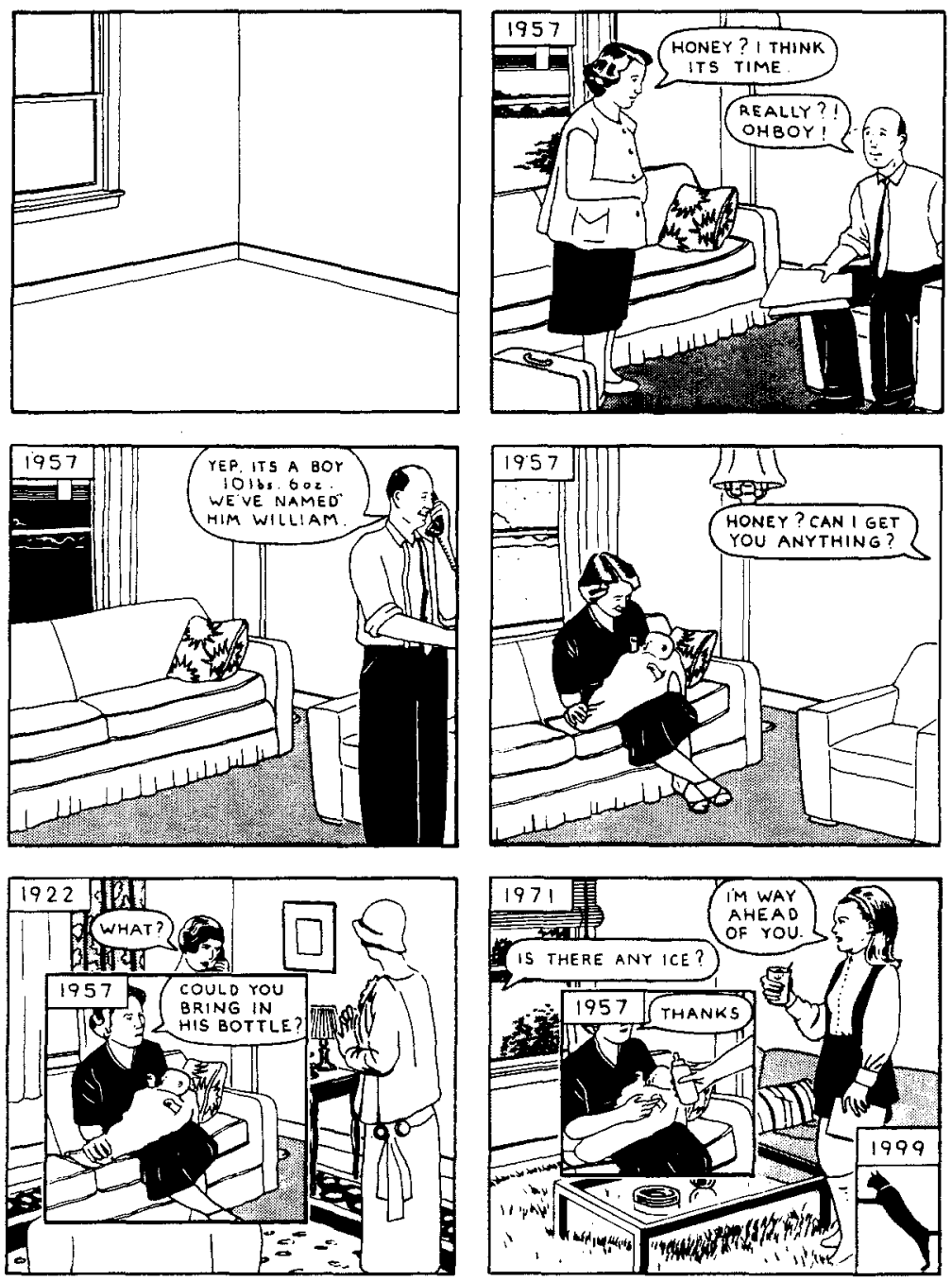

Figure 1 

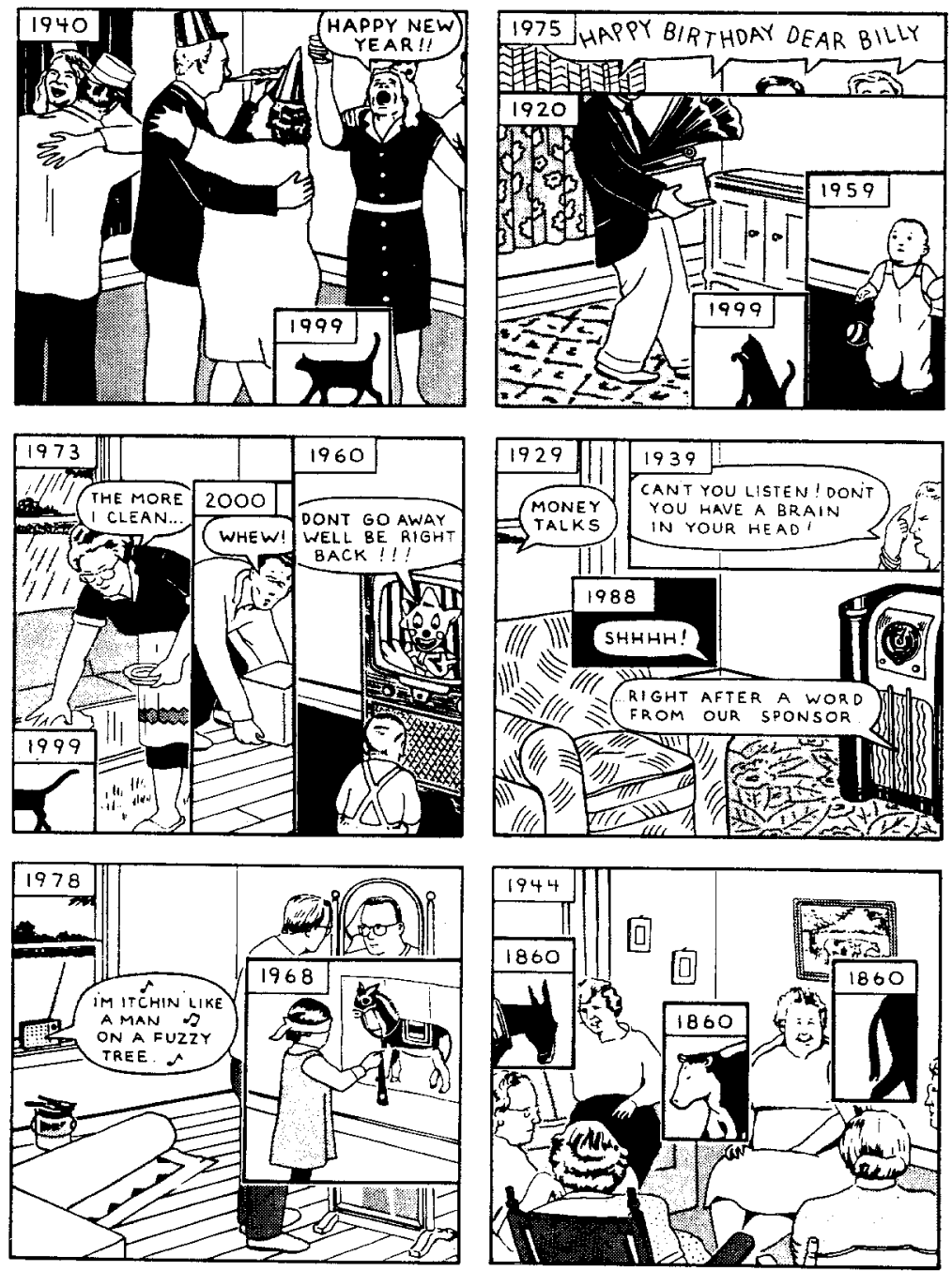

Figure 2 

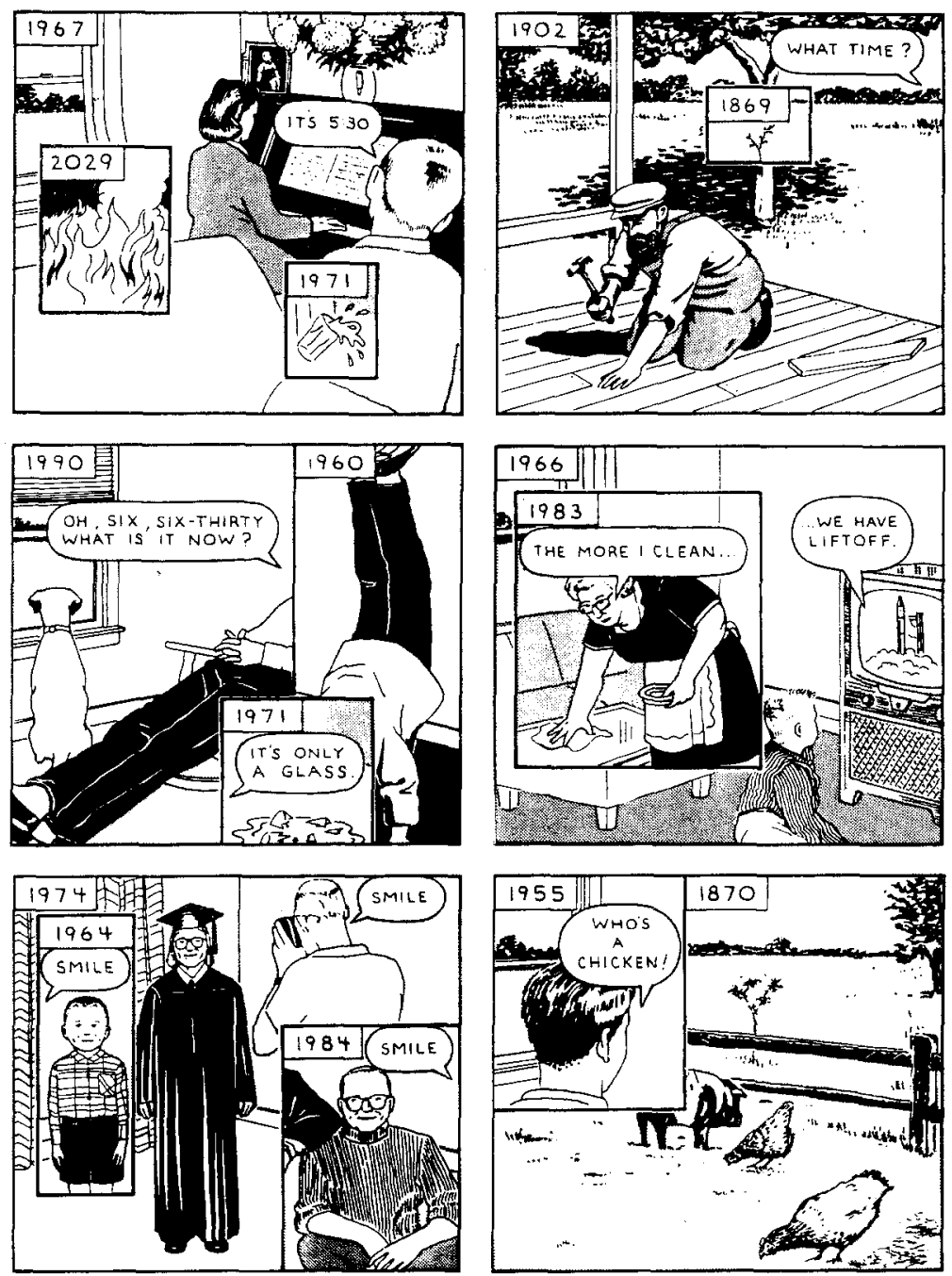

Figure 3 

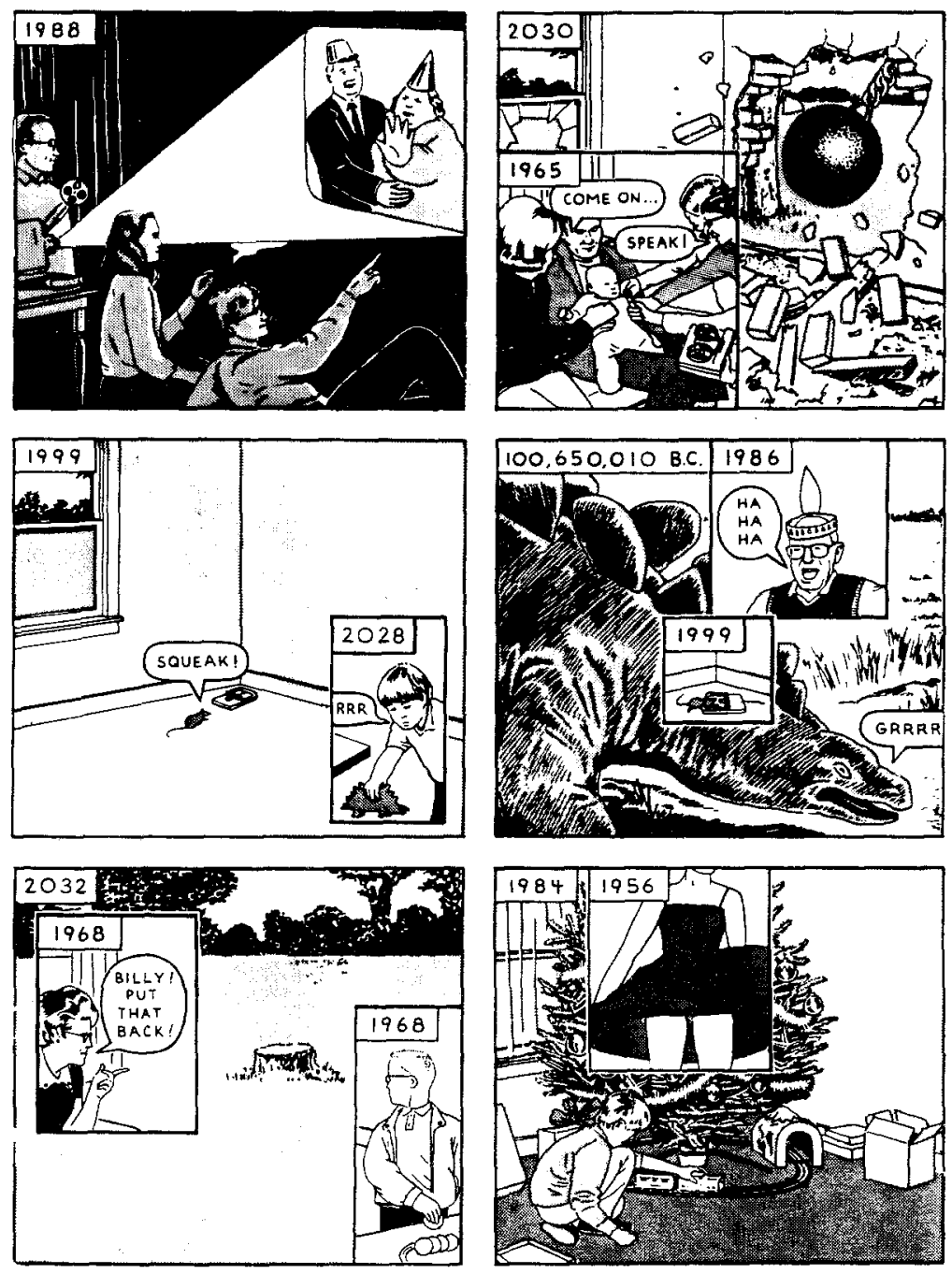

Figure 4 

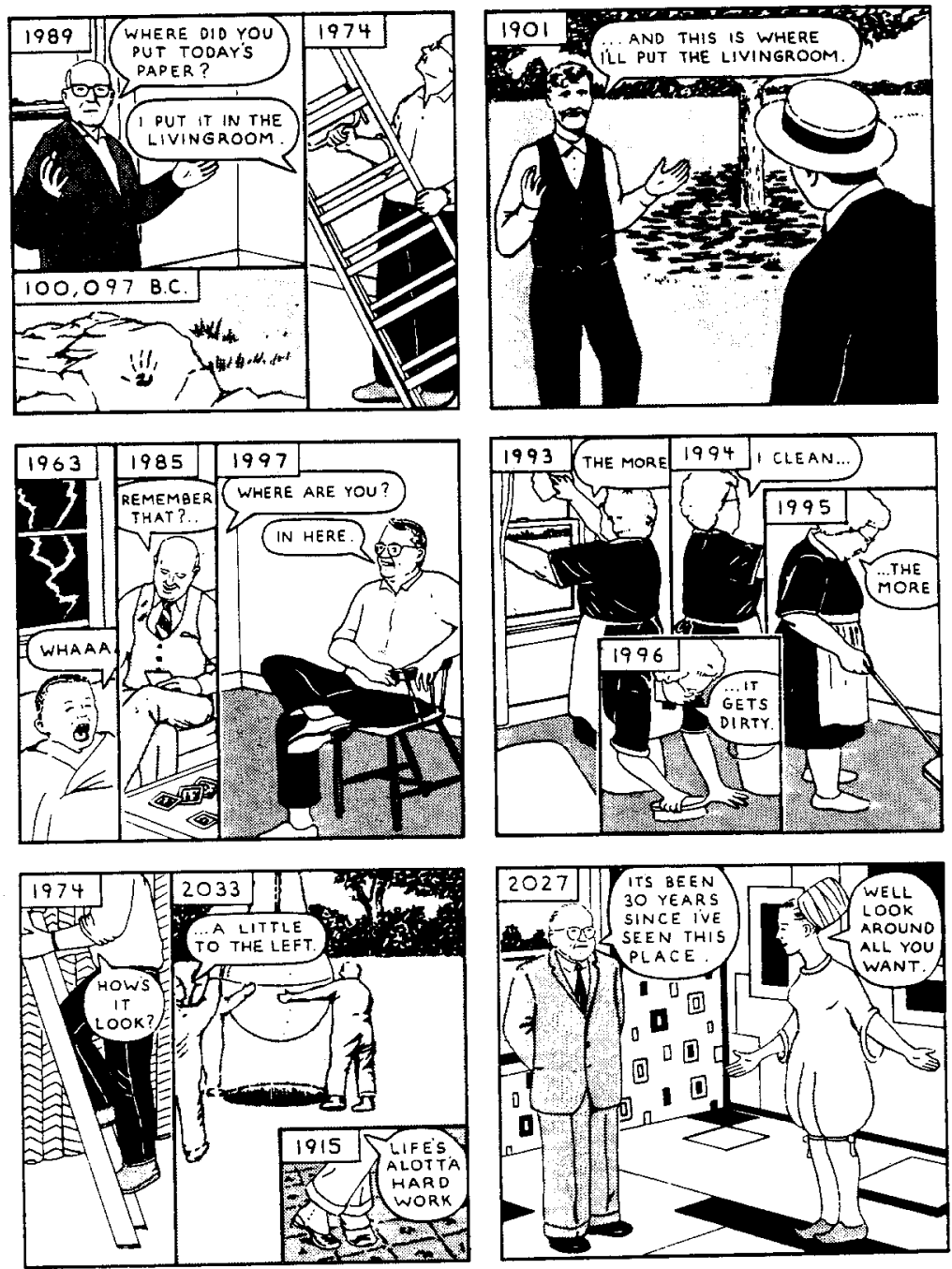

Figure 5 

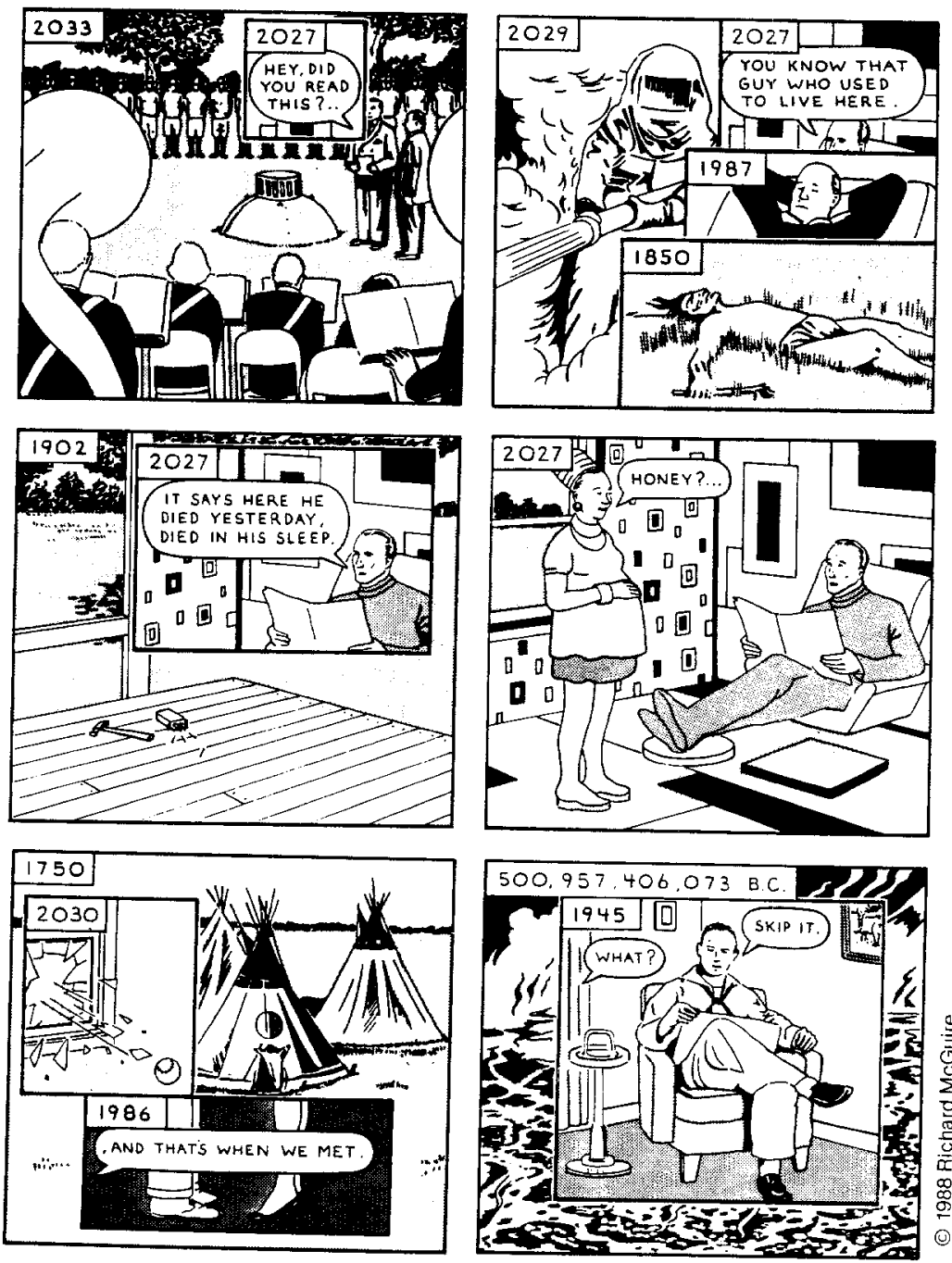

Figure 6 